\title{
Pharmacologic Effect of Miao Medicine Illicium simonsii Maxim. on Collagen-Induced Arthritis in Rats
}

\author{
Qing-zhong Zhang $\mathbb{D}^{\mathbb{B}}$, Xian-yun Hu, Wen-qin Yao, Xiao-li Wan, Zi-cong Liang, \\ Rong-min Dang, Zheng-min Yang, and Ya-lan Xia
}

Qiannan Medicine College for Nationalities, Duyun, Guizhou 558000, China

Correspondence should be addressed to Qing-zhong Zhang; zhangqingzhong@qq.com

Received 8 August 2018; Revised 20 September 2018; Accepted 26 September 2018; Published 11 October 2018

Academic Editor: Armando Zarrelli

Copyright (c) 2018 Qing-zhong Zhang et al. This is an open access article distributed under the Creative Commons Attribution License, which permits unrestricted use, distribution, and reproduction in any medium, provided the original work is properly cited.

Objectives. To study the pharmacologic effect and mechanism of action of Miao medicine Illicium simonsii Maxim. (ISM) in treating rheumatoid arthritis. Methods. Sixty rats were randomly divided to six groups: normal control (normal), collagen-induced arthritis (CIA) model (model), CIA + tripterygium glycosides (TG), CIA + ISM high dose oral (ISM-H), CIA + ISM low-dose oral (ISML), and CIA + ISM topical application (ISM-T). The treatment doses were selected based on published reports and folk medicine practice. The outcome measurements included paw swelling, joint pathology, organ index, blood count, Thelper 17 (Th17) cell count, and interleukin-6 (IL-6) level. Results. Compared to the CIA model group, all treatment groups showed a significant reduction in paw swelling, blood vessel pathology, Th17 cell count, and IL-6 levels $(p<0.05$ or $p<0.01)$. All treatment groups showed alleviated foot swelling and lower total number of white blood cells, and these effects were observed earlier with oral ISM than topical ISM. The effect of ISM was weaker than that of TG. In addition, less organ damage was observed with topical ISM than oral ISM but better than TG. Conclusions. These results suggest that, by downregulating Th17 cells, ISM inhibits the production of Il-6, thereby alleviating the proliferation of endothelial and rheumatoid-like cells and leukocytosis in CIA rats, ultimately eliminating foot swelling.

\section{Introduction}

Rheumatoid arthritis (RA) is a chronic systemic disease characterized by inflammatory synovitis that mainly involves small joints of the hands and feet. RA is often accompanied by abnormalities involving extra-articular organs, joint deformity, and loss of function, thus negatively impacting physical and mental health, daily life, and economic status. Its etiology remains unclear. Approximately $0.3 \%$ and $0.5 \%-1 \%$ of the Chinese and global populations, respectively, are affected by RA [1-3]. Approximately $35 \%$ of patients with uncontrolled RA lose their ability to work within 10 years $[4,5]$.

Advances in nanotechnology, chemical synthesis, and enzymology [6-8] have extensively improve RA treatment. However, these have not been utilized in Chinese clinics due to the limiting factors such as inadequate medical facilities and their associated high cost. Compared to RA medicines such as tripterygium glycosides and methotrexate, there are several domestic drugs in China that are effective in treating
RA $[9,10]$. However, their application is limited because of their toxicity and side effects.

In Guizhou Miao medicine, people have traditionally employed Illicium simonsii Maxim. (ISM) to alleviate RA by either oral or topical application [11-15]. While some studies have conducted chemical and toxicological analyses of ISM $[12,13,16,17]$, no investigation on its therapeutic efficacy in RA has been conducted. The present study established a collagen-induced arthritis (CIA) rat model to determine the therapeutic efficacy of ISM on RA development and its effect on serum IL-6 levels, Th17 cell count [18], and synovial MMP13 expression [19]. The results will help in establishing the medicinal applications of ISM.

\section{Materials and Methods}

2.1. Preparation of ISM. Dried ISM fruits were purchased from the Miao Region in Guizhou Province and authenticated by Prof. Xuejun Wei at the Qiannan Medical College 
for Nationalities (Duyun, Guizhou, China). ISM (5,000 g) was extracted thrice (30 min each time) using reflux extraction, and the resulting liquid was pooled. After rotary evaporation, $1,750 \mathrm{~g}$ of extract was obtained.

2.2. Animals and Reagents. Sixty specific pathogen-free male Sprague-Dawley $(\mathrm{SD})$ rats $(180 \pm 20 \mathrm{~g})$ were purchased from the Laboratory Animal Center of the Third Military Medical University, a center certified by the China Ministry of Public Health. The tripterygium glycosides were from the Deende Pharmaceutical, Inc. (Zhejiang, China). Complete Freund's adjuvant (CFA) was purchased from Sigma (Shanghai Greater China, China); IL-6, IL-17, and bovine collagen type II were purchased from Solarbio Science and Technology (Beijing, China), Wilking Biological Technology (Nanning, China), and Dingshengtai Biotechnology (Guizhou, China), respectively.

\subsection{Animal Model Establishment and Drug Administration.} The 60 SD male rats were randomly divided into six groups (10 rats/group), which included a normal control group and five CIA disease model groups. All experiments were conducted following the animal welfare and protection regulations. Type II bovine collagen was dissolved in acetic acid $(0.05 \mathrm{~mol} / \mathrm{L})$ to make a $2 \mathrm{mg} / \mathrm{mL}$ solution, which was incubated with shaking at $4^{\circ} \mathrm{C}$ overnight. Equal volumes of type II bovine collagen solution and CFA were mixed to make an emulsion, of which $0.1 \mathrm{~mL}$ was injected to the right hind food pad of each rat to induce CIA. The CIA rats were divided into five treatment groups (10 rats/group): CIA model (model), CIA + tripterygium glycosides (TG), CIA + ISM high-dose oral (ISM-H), CIA + ISM low-dose oral (ISM-L), and CIA + ISM topical application (ISM-T). Approximately 10 days after CIA induction, the rats received their respective treatments. The rats in the normal control group (normal) were given saline via gavage, and those in the ISM-T group were treated externally using their right hind foot and ankle joint. The rats in the TG treatment group were given a daily gavage of tripterygium glycosides at a dose of $50 \mathrm{mg} / \mathrm{kg}$ body weight [20]. The rats in oral ISM high-dose (ISM-H) and lowdose (ISM-L) groups were given a daily gavage of $2.1 \mathrm{~g}$ and $0.7 \mathrm{~g} / \mathrm{kg}$ body weight, respectively. These oral doses of ISM were selected based on folk medicine practice and reported pharmacotoxicology results [14]. The rats in the ISM-T group were given ISM daily at a dose of $3.5 \mathrm{~g} / \mathrm{kg}$ body weight by local application to the right hind foot and ankle joints after shaving the treatment site to remove any hair.

2.4. Foot Swelling. At $-1,3,7 \ldots \ldots 27$ days (once every four days) after treatment, swelling of the right hind foot joints was measured with a caliper.

2.5. Organ Indices. The day after the last drug administration, the rats were anesthetized by peritoneal injection of $10 \%$ chloral hydrate $(0.1 \mathrm{~mL} / 100 \mathrm{~g} \mathrm{~kg}$ body weight $)$ [19] and then euthanized by cervical dislocation. The spleen, kidneys, liver, lungs, and thymus were collected and weighed for organ index calculation.
2.6. Serum IL-6 Analysis by ELISA. Blood ( $2.5 \mathrm{~mL}$ ) was collected after the rats were euthanized. Blood was left to stand for $1 \mathrm{~h}$ and then centrifuged at 2,000 rpm for $15 \mathrm{~min}$ to obtain the serum. Serum IL- 6 was quantified using an ELISA kit following the manufacturer's instructions.

2.7. Measurement of Th17 Cells by Flow Cytometry. After the rats were euthanized as earlier described, $250 \mu \mathrm{L}$ of whole blood was collected and diluted with RPMI 1640 medium at a ratio of $1: 1$, resulting in a total volume of $500 \mu \mathrm{L}$. Approximately $2 \mu \mathrm{L}$ of a stimulator/blocker solution was added to the diluted blood and then incubated at $37^{\circ} \mathrm{C}$ in $5 \% \mathrm{CO}_{2}$ for $4 \mathrm{~h}$. After centrifugation and removal of the supernatant, red blood cell lysis buffer was added $(2 \mathrm{~mL} / 100 \mu \mathrm{L}$ whole blood), and the mixture was incubated in the dark for $10 \mathrm{~min}$. Then, the samples were centrifuged to remove the supernatant, and then $5 \mu \mathrm{L}$ of FITC-labeled anti-CD4 was added and thoroughly mixed. After incubation in the dark at $2-8^{\circ} \mathrm{C}$ for $15 \mathrm{~min}, 2 \mathrm{~mL}$ of a staining buffer was added to resuspend the cells, followed by centrifugation at $1,200 \mathrm{rpm}$ for $5 \mathrm{~min}$. After removing the supernatant, $250 \mu \mathrm{L}$ of a Fix/Perm buffer was added, mixed, and then incubated in the dark at $2-8^{\circ} \mathrm{C}$ for $20 \mathrm{~min}$, at which an additional $1 \mathrm{~mL}$ of $1 \times$ Perm/Wash buffer was added and mixed. After centrifugation, part of the supernatant was removed, leaving $250 \mu \mathrm{L}$ of liquid, upon which $1 \mathrm{~mL} 1 \times$ Perm/Wash buffer was added to resuspend the cells. After another centrifugation at $1,200 \mathrm{rpm}$ for $5 \mathrm{~min}$, the supernatant was removed and $80 \mu \mathrm{L}$ to $100 \mu \mathrm{L}$ of the stain buffer was added to resuspend cells, to which $5 \mu \mathrm{L}$ of PElabeled IL-17A antibody was added, mixed, and incubated in the dark for $30 \mathrm{~min}$. Next, $2 \mathrm{~mL}$ of $1 \times$ Perm/Wash buffer was added to resuspend the cells, which were again centrifuged at $1,200 \mathrm{rpm}$ for $5 \mathrm{~min}$, and then the supernatant was removed. Finally, $500 \mu \mathrm{L}$ of the stain buffer was added to resuspend the cells, which were then passed through a $300-\mu \mathrm{m}$ filter, followed by flow cytometry analysis.

2.8. HE Staining of Foot Joints. After the rats were euthanized, the foot joints were collected and fixed with $4 \%$ paraformaldehyde, followed by a series of processes, including fixation, decalcification, deacidification, dehydration, embedding, and sectioning. The tissue sections were examined under a microscope.

2.9. Blood Tests. After the rats were euthanized, $3 \mathrm{~mL}$ whole blood was collected for routine blood testing.

2.10. Statistical Analysis. The measurement data were expressed as mean \pm standard deviation $(\overline{\mathrm{X}} \pm \mathrm{SD})$. The differences among groups were analyzed by one-way ANOVA, and the LSD test was used for pairwise comparisons. $p<0.05$ was considered statistically significant.

\section{Results}

3.1. Foot Swelling. Table 1 shows that, compared to the CIA model group, the normal group (from day 3 ), the TG, ISM$\mathrm{H}$, and ISM-L oral groups (all from day 19), and ISM-T 
TABLE 1: Rat foot swelling (mm).

\begin{tabular}{lcccccc}
\hline Time $(\mathrm{d})$ & Normal & Model & TG & ISM-H & ISM-L & ISM-T \\
\hline-1 & $4.73 \pm 1.08$ & $14.79 \pm 0.43$ & $4.81 \pm 0.79$ & $4.74 \pm 0.65$ & $4.68 \pm 0.51$ & $7.85 \pm 0.64$ \\
3 & $5.02 \pm 1.26^{* *}$ & $7.47 \pm 2.45^{* *}$ & $7.58 \pm 2.56^{* *}$ & $7.63 \pm 2.61^{* *}$ & $7.50 \pm 2.48^{* *}$ & $7.66 \pm 2.65^{* *}$ \\
7 & $5.22 \pm 1.18^{* *}$ & $7.50 \pm 0.08$ & $7.69 \pm 1.38$ & $7.50 \pm 1.34$ & $7.45 \pm 0.68$ & $7.74 \pm 1.07$ \\
11 & $5.52 \pm 0.89^{* *}$ & $7.88 \pm 1.53$ & $7.76 \pm 1.05$ & $7.81 \pm 0.98$ & $8.01 \pm 1.08$ & $7.91 \pm 1.28$ \\
15 & $5.78 \pm 0.58^{* *}$ & $7.82 \pm 1.19$ & $7.69 \pm 1.09$ & $7.70 \pm 0.68$ & $7.75 \pm 1.05$ & $7.69 \pm 1.08$ \\
19 & $6.03 \pm 0.74^{* *}$ & $7.65 \pm 1.08$ & $6.89 \pm 0.98$ & $7.03 \pm 0.91^{*}$ & $7.09 \pm 0.87^{*}$ & $7.50 \pm 1.02$ \\
23 & $6.15 \pm 1.02^{* *}$ & $7.41 \pm 1.01$ & $6.36 \pm 0.75^{* *}$ & $6.81 \pm 0.78^{* * \#}$ & $7.06 \pm 1.01^{* \# \#}$ & $7.20 \pm 0.88^{\#}$ \\
27 & $6.28 \pm 1.19^{* *}$ & $7.15 \pm 1.57$ & $6.21 \pm 0.88^{* *}$ & $6.55 \pm 0.58^{* *}$ & $6.35 \pm 0.73^{* *}$ & $6.66 \pm 0.62^{*}$ \\
\hline Note: ${ }^{*} p<0.05,{ }^{* *} p<0.01$, vs model group ${ }^{\#} p<0.05,{ }^{\# \#} p<0.01$, vs TG group. & & &
\end{tabular}

TABLE 2: Rat organ index.

\begin{tabular}{|c|c|c|c|c|c|}
\hline Group & Spleen & Kidney & Liver & Lung & Thymus \\
\hline Normal & $0.19 \pm 0.08$ & $0.73 \pm 0.01$ & $3.71 \pm 0.31$ & $0.51 \pm 0.12^{*}$ & $0.12 \pm 0.05$ \\
\hline Model & $0.25 \pm 0.03$ & $0.73 \pm 0.01$ & $4.14 \pm 0.81$ & $0.56 \pm 0.04$ & $0.16 \pm 0.02$ \\
\hline TG & $0.18 \pm 0.01^{*}$ & $0.73 \pm 0.02$ & $3.73 \pm 0.39$ & $0.46 \pm 0.07^{* *}$ & $0.12 \pm 0.08^{*}$ \\
\hline ISM-H & $0.23 \pm 0.02^{\# @ ~}$ & $0.74 \pm 0.03$ & $4.49 \pm 0.39^{\# \# @ ~}$ & $0.52 \pm 0.06^{\# @ ~}$ & $0.14 \pm 0.01$ \\
\hline ISM-L & $0.23 \pm 0.06^{\# \#}$ & $0.71 \pm 0.03$ & $4.04 \pm 0.48$ & $0.50 \pm 0.06$ & $0.14 \pm 0.01$ \\
\hline ISM-T & $0.20 \pm 0.02$ & $0.73 \pm 0.07$ & $3.93 \pm 0.77^{\# \#}$ & $0.49 \pm 0.09^{* *}$ & $0.13 \pm 0.04$ \\
\hline
\end{tabular}

Note: ${ }^{*} p<0.05,{ }^{* *} p<0.01, v s$. model group; ${ }^{\#} p<0.05,{ }^{\# \#} p<0.01, v s$. TG group; ${ }^{@} p<0.05, v s$. ISM-T group.

group (from day 27) all exhibited significant attenuation of foot swelling $(p<0.05$ or $p<0.01)$. No significant difference between each administration group and TG group (except on the 23rd day), as well as between the ISM-T group and the ISM-oral group $(p>0.05)$, was observed.

3.2. Organ Indices. Table 2 shows that, compared to the model group, the normal group, the TG group, and the ISM-T group had significantly lower lung index $(p<0.05$ or $p<0.01)$, and the spleen and thymus indexes were also significantly lower in the TG group $(p<0.05)$. Compared to the TG group, the ISM-H and ISM-L groups had higher spleen index $(p<$ 0.05), the ISM-H group and ISM-T group had higher liver index $(p<0.01)$, and the ISM-H group had higher lung index $(p<0.05)$. Compared to the ISM-T group, the ISM-H group had higher spleen, liver, and lung indexes $(p<0.05)$.

3.3. Serum IL-6 Levels. Figure 1 shows that, compared to the CIA model group, the normal group, the TG group, the ISM$\mathrm{H}$ and ISM-L groups, and the ISM-T group had significantly lower serum IL- 6 levels $(p<0.05$ or $p<0.01)$. This reduction was greater in the ISM-H group compared to the TG group $(p<0.05)$.

3.4. Th17 Cells. Figure 2 shows the Th17 populations in different groups as determined by flow cytometry analysis. Compared to the CIA model group, the percentage of Th17 cells in the total number of lymphocytes was significantly lower in all the other groups $(p<0.05$ or $p<0.01)$. No significant difference between the ISM treatment groups and the TG group, or between the ISM oral groups and the topical group $(p>0.05)$, was observed (Figure 3 ).
3.5. Pathology (HE Staining). Figure 4 shows the pathological sections of the joints. The blood vessels in the feet of the CIA model rats showed hyperplasia in rheumatoid-like cells, endothelial cells, epithelial cells, and fibrous tissues (Figure 4). Based on the joint pathological scoring method [21], the rats in the ISM-H and ISM-L groups, ISM-T groups, and the TG group all showed significant improvement in reducing hyperplasia in blood vessel fibrous tissues, endothelial cells, and rheumatoid-like cells $(p<0.05$ or $p<0.01)$. The effect on the ISM-H, ISM-L, and ISM-T groups in reducing hyperplasia of rheumatoid-like cells, endothelial cells, epithelial cells, and fibrous tissues was less significant than that in the TG group $(p<0.05$ or $p<0.01)$. Compared to the ISM-T group, the ISM-H group showed more extensive improvement in reducing endothelial cell proliferation $(p<$ 0.05 ), and the ISM-H and ISM-L groups showed more significant improvement in reducing the number of rheumatoidlike cells $(p<0.05$ or $p<0.01)$ (Table 3$)$.

3.6. Blood Test. Compared to the CIA model group, the normal, TG, ISM-H, and ISM-L groups had significantly fewer total white blood cells (WBCs) $(p<0.05$ or $p<0.01)$. The normal, TG, ISM-H, and ISM-L groups had significantly fewer monocytes $(p<0.05$ or $p<0.01)$. The ISM-H and ISM$\mathrm{T}$ groups had more eosinophils $(p<0.05$ or $p<0.01)$. The TG group had fewer total platelet counts, whereas the ISMT group had more $(p<0.05)$. Compared to the TG group, the ISM-T group had more neutrophils $(p<0.05)$, the ISM$\mathrm{H}, \mathrm{ISM}-\mathrm{L}$, and ISM-T groups had more monocytes $(p<0.05$ or $p<0.01$ ), and the ISM-H and ISM-T groups had more eosinophils $(p<0.05$ or $p<0.01)$ and total platelet counts $(p$ $<0.01$ ) (Table 4). 


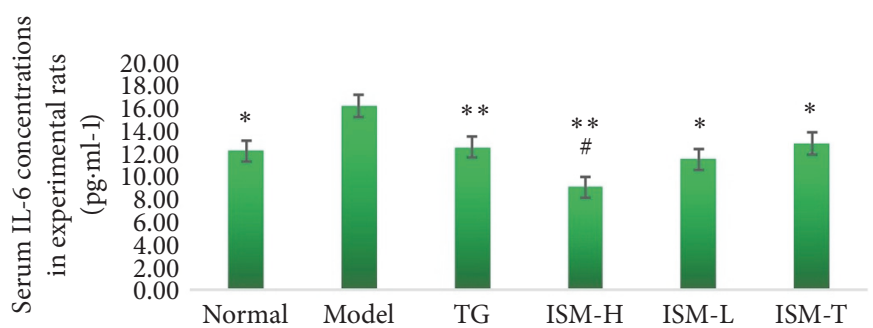

Figure 1: Serum IL-6 concentrations in experimental rats. Note: ${ }^{*} p<0.05,{ }^{* *} p<0.01$, vs model group; ${ }^{\#} p<0.05$, vs TG group.
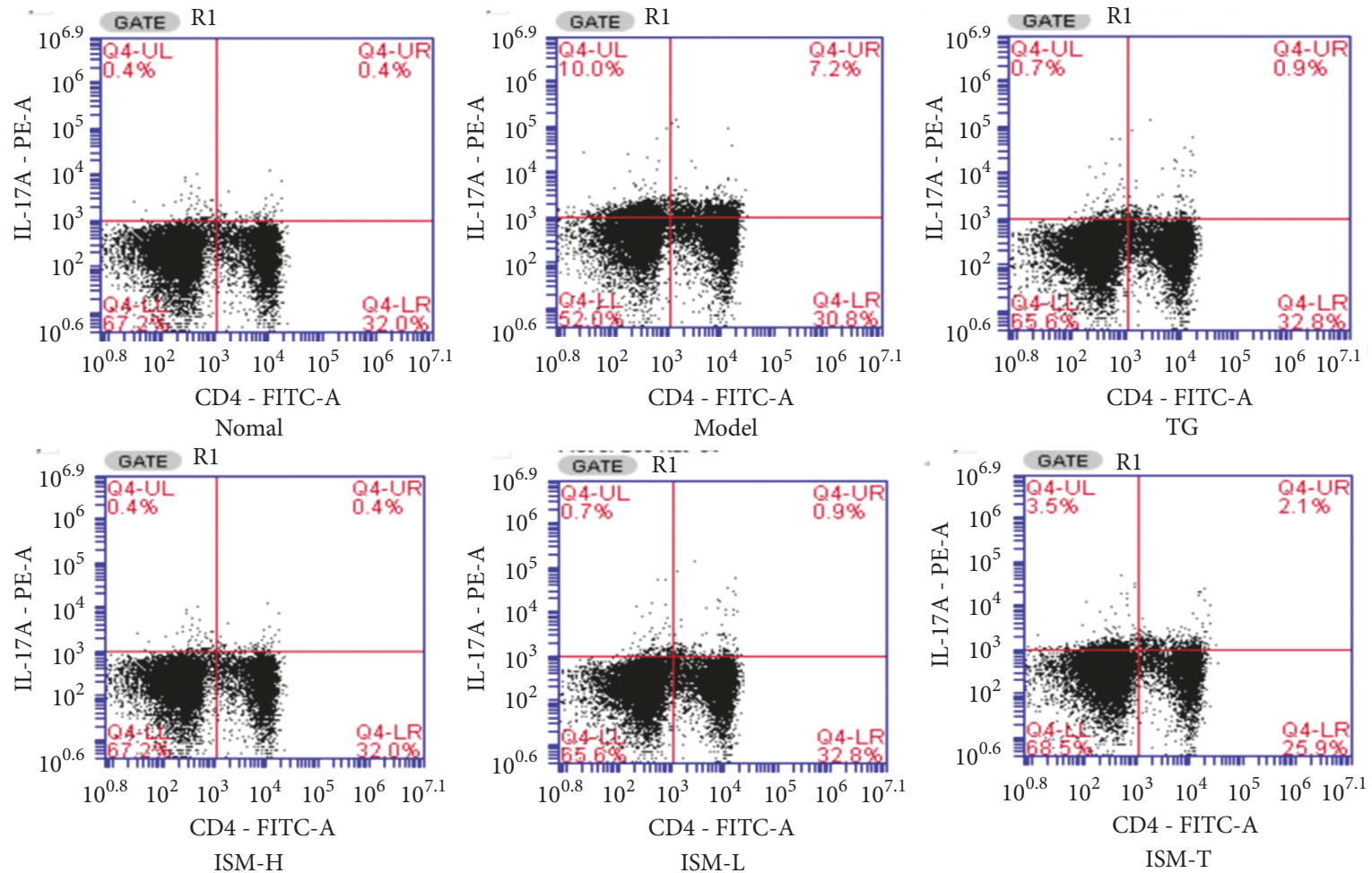

FIGURE 2: Flow cytometry analysis of Th17 cells in the experimental rats.

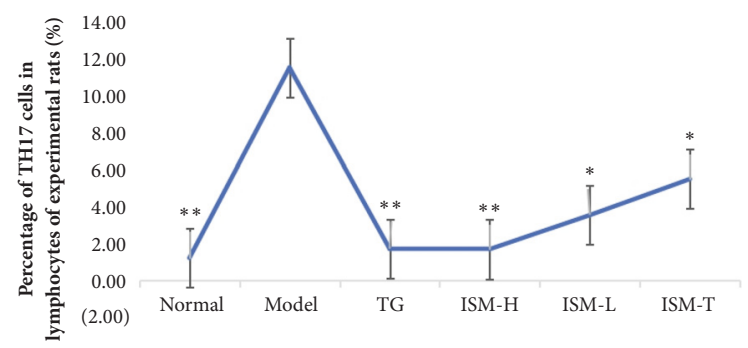

FIGURE 3: Percentage of Th17 cells in the lymphocytes of the experimental rats. Note: ${ }^{*} p<0.05,{ }^{* *} p<0.01$, vs model group.

\section{Discussion}

4.1. Pharmacological Efficacy, Dose, and Effect of ISM on Organs. Comparison of foot swelling among different treatment groups (Table 1) showed that the well-recognized antiRA drug tripterygium glycoside and both oral and topical
ISM administration could significantly improve foot swelling, and this effect was not significantly different among treatments, except that the effects of ISM were observed later when topically administered. These results indicate that ISM is effective in improving foot swelling in CIA rats, and oral administration is superior to topical application. Furthermore, this effect of ISM is similar to the well-recognized anti-RA drug tripterygium glycoside.

Table 2 shows that the spleen, liver, and lung indices were higher with topical ISM than oral, and the spleen, liver, and lung indices downward effects of ISM, regardless of administration, were significantly better than that using tripterygium glycosides. These results showed that ISM is more effective in the treatment of CIA in rats than tripterygium glycosides.

Pathological scores of the joint sections (Table 3) demonstrated that ISM application significantly alleviated toe vascular fibrosis and improved endothelial cell proliferation and the number of rheumatic-like cells in the CIA rats. ISM oral administration has significantly stronger effectiveness than 


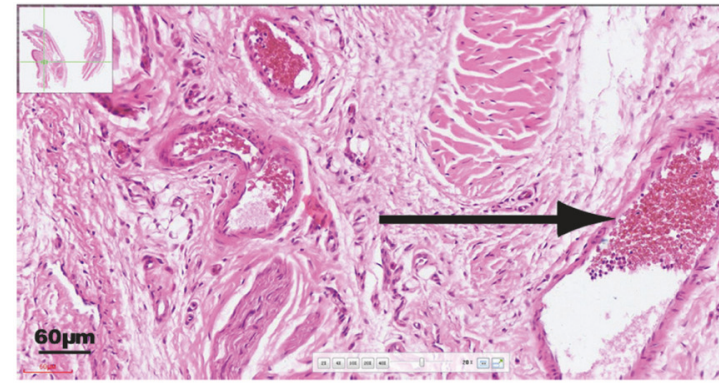

Normal

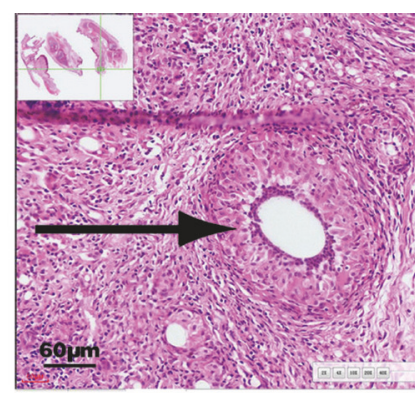

Model

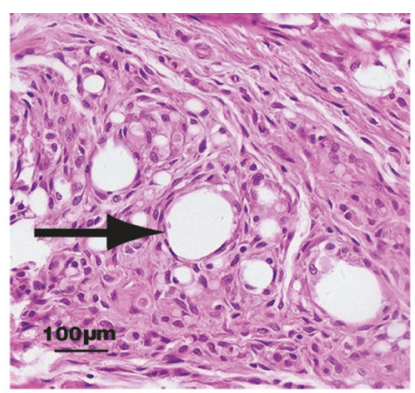

TG

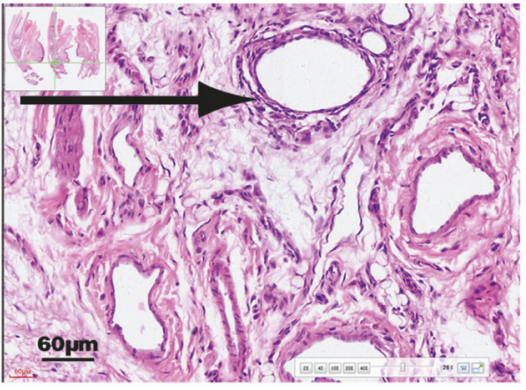

ISM-H

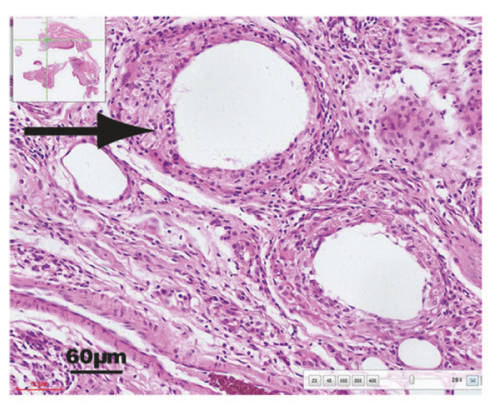

ISM-L

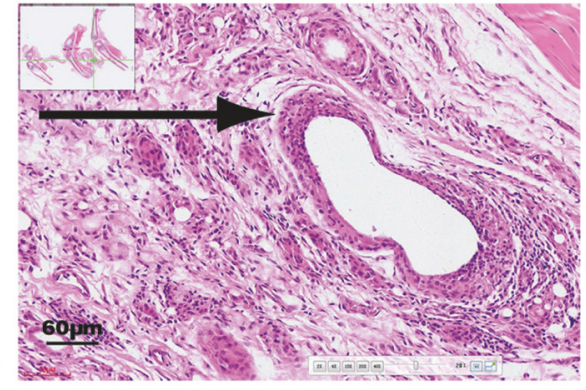

ISM-T

Figure 4: Pathology of rat foot and ankle joints (HE staining, 20×).

TABLE 3: Rat foot joint pathological scores.

\begin{tabular}{lccc}
\hline Group & Blood vessel fibrosis & Endothelial proliferation & Rheumatoid-like cells \\
\hline Normal & 0 & 0 & 0 \\
Model & $1.66 \pm 0.09$ & $2.45 \pm 0.05$ & $1.14 \pm 0.03$ \\
TG & $0.49 \pm 0.07^{* *}$ & $0.80 \pm 0.04^{* *}$ & $0.22 \pm 0.03^{* *}$ \\
ISM-H & $1.18 \pm 0.02^{* * \# \#}$ & $2.13 \pm 0.05^{* * \# @}$ & $0.70 \pm 0.07^{* * \# @ @}$ \\
ISM-L & $1.38 \pm 0.17^{* * \# \#}$ & $2.22 \pm 0.02^{* * \# \#}$ & $0.90 \pm 0.04^{* * \# \# @}$ \\
ISM-T & $1.41 \pm 0.09^{* * \# \#}$ & $2.34 \pm 0.01^{* * \# \#}$ & $1.10 \pm 0.07^{* \# \#}$ \\
\hline
\end{tabular}

Note: ${ }^{*} p<0.05,{ }^{* *} p<0.01$, vs model group; ${ }^{\# \#} p<0.01$, vs TG group; ${ }^{@} p<0.05,{ }^{@ @ ~} p<0.01$, vs ISM-T group.

TABLE 4: Rat blood test.

\begin{tabular}{lcccccc}
\hline Group & $\begin{array}{c}\text { Total white blood } \\
\text { cell count }\end{array}$ & $\begin{array}{c}\text { Percentage of } \\
\text { neutrophils }\end{array}$ & $\begin{array}{c}\text { Percentage of } \\
\text { lymphocytes }\end{array}$ & $\begin{array}{c}\text { Percentage of } \\
\text { monocytes }\end{array}$ & $\begin{array}{c}\text { Percentage of } \\
\text { eosinophils }\end{array}$ & $\begin{array}{c}\text { Total platelet count } \\
\text { Normal }\end{array}$ \\
Model & $4.25 \pm 0.37^{* *}$ & $0.20 \pm 0.02$ & $0.76 \pm 0.11$ & $0.06 \pm 0.01^{* *}$ & $0.03 \pm 0.01$ & $297.00 \pm 0.98$ \\
TG & $2.82 \pm 0.27$ & $0.34 \pm 0.06$ & $0.58 \pm 0.09$ & $0.21 \pm 0.04$ & $0.06 \pm 0.02$ & $266.50 \pm 1.38$ \\
ISM-H & $3.50 \pm 0.67^{*}$ & $0.18 \pm 0.05$ & $0.74 \pm 0.10$ & $0.09 \pm 0.05^{*}$ & $0.06 \pm 0.01$ & $186.50 \pm 1.35^{*}$ \\
ISM-L & $3.30 \pm 0.56^{*}$ & $0.14 \pm 0.02^{@}$ & $0.72 \pm 0.08$ & $0.15 \pm 0.02^{* \#}$ & $0.09 \pm 0.03^{* \# @}$ & $424.50 \pm 2.30^{\#}$ \\
ISM-T & $3.94 \pm 0.36$ & $0.23 \pm 0.03$ & $0.70 \pm 0.06$ & $0.12 \pm 0.03^{* \# @ @}$ & $0.06 \pm 0.01^{@ @ ~}$ & $243.00 \pm 1.76^{\# @ @ ~}$ \\
\hline
\end{tabular}

Note: ${ }^{*} p<0.05,{ }^{* *} p<0.01$, vs model group; ${ }^{\#} p<0.05,{ }^{\# \#} p<0.01, v s$. TG group; ${ }^{\circledR} p<0.05$, ${ }^{@} p<0.01, v s$. ISM-T group.

topical use, but weaker than tripterygium glycosides. However, ISM has fewer side effects than tripterygium glycosides.

Table 4 shows that both ISM oral and topical administration can cut down the total number of leukocytes and monocytes in rats, but the reduction was more pronounced with oral ISM, and the percentage of eosinophils and the total number of platelets in the topical ISM group significantly increased. The effect of the oral ISM administration is better than topical administration and did not significantly differ from the well-recognized anti-RA drug tripterygium glycoside.

4.2. Mechanistic Study of the Anti-RA Effect of ISM. IL-6 is an important cytokine derived from monocytes/macrophages, and its interaction with several other cytokines is essential to the induction of RA $[18,22,23]$. Th17 cells produce IL-17, 
which is an inflammatory cytokine that has potent proinflammatory activity and works with other cytokines to amplify inflammatory responses $[24,25]$. IL-17 can stimulate the production of IL- 6 and monocyte chemoattractant protein1 (MCP-1) by synovial cells, fibroblasts, and endothelial cells $[25,26]$. The present study observed that ISM, through both oral and topical administration, significantly reduced IL-6 levels (Figure 1) and the number of Th17 cells (Figure 3). Assessment of foot swelling, toe histopathological sections, and blood routine studies indicate that the IL-6 levels and the number of Th17 cells are positively correlated with foot swelling, cell proliferation, and the increase of leukocyte count, which agrees with the results of a previous study [27]. Therefore, by downregulating Th17 cells, ISM may inhibit the production of a variety of cytokines such as Il-6 [25, 26], reduce endothelial cells to express adhesion molecules, and consequently decrease monocyte migration to the inflammation sites $[28,29]$, and then inhibit multiple cellular responses [cell multiplication, cell differentiation, enhancement of immune response, and angiogenesis [18]] to alleviate the proliferation of endothelial cells and rheumatoid-like cells and leukocytosis in CIA rats. In addition, ISM is superior to tripterygium glycosides for the treatment of CIA.

\section{Conclusions}

ISM is a Miao medicine that is commonly used for the treatment of RA. ISM is widely distributed in China, has few side effects, and can be used both orally and topically. By downregulating Th17 cells, ISM inhibits the production of Il- 6 and reduces the expression of adhesion molecules in endothelial cells, consequently decreasing monocyte migration to the inflammation sites, alleviating the proliferation of endothelial and rheumatoid-like cells and leukocytosis, ultimately eliminating foot swelling in CIA rats.

\section{Data Availability}

The data used to support the findings of this study are available from the corresponding author upon request.

\section{Conflicts of Interest}

The authors have no conflicts of interest to declare.

\section{Acknowledgments}

The authors thank the Department of Pharmacology, Zunyi Medical School, for assisting in measuring foot swelling, assessment of organ indices, and IL-6 analyses; the Department of Pathology and Clinical Lab of Zunyi Medical School Hospital for conducting pathology and blood tests, respectively; and the Nanning Weierkai Bio Tech, Inc., for conducting Th17 analysis. We also thank Dr. Jie Liu for his advice throughout the study. The Basic Research Projects of the Science and Technology Office, Guizhou Province, China (2017-1170), the Science and Technology Office, Qiannan City, Guizhou Province, China (2016-6), and a grant from the Qiannan Medical School of Nationalities (QNYZ2016-10) supported this study.

\section{References}

[1] D. T. Felson, "Comparing the prevalence of rheumatoid diseases in china with the rest of the world," Arthritis Research \& Therapy, vol. 10, no. 1, 2008.

[2] T. Uhlig, R. H. Moe, and T. K. Kvien, "The burden of disease in rheumatoid arthritis," PharmacoEconomics, vol. 32, no. 9, pp. 841-851, 2014.

[3] C. Xu, X. Wang, R. Mu et al., "Social costs of rheumatoid arthritis in China: a hospital-based cross-sectional study," Arthritis Care \& Research, vol. 66, no. 4, pp. 523-531, 2014.

[4] S. Allaire, F. Wolfe, J. Niu, and M. P. Lavalley, "Contemporary prevalence and incidence of work disability associated with rheumatoid arthritis in the US," Arthritis Care \& Research, vol. 59, no. 4, pp. 474-480, 2008.

[5] H. Radner, T. Neogi, J. S. Smolen, and D. Aletaha, "Performance of the 2010 ACR/EULAR classification criteria for rheumatoid arthritis: a systematic literature review," Annals of the Rheumatic Diseases, vol. 73, no. 1, pp. 114-123, 2013.

[6] S.-M. Lee, H. J. Kim, Y.-J. Ha et al., "Targeted chemo-photothermal treatments of rheumatoid arthritis using gold half-shell multifunctional nanoparticles," ACS Nano, vol. 7, no. 1, pp. 5057, 2013.

[7] M. J. Kang, Y.-J. Park, S. You et al., "Urinary proteome profile predictive of disease activity in rheumatoid arthritis," Journal of Proteome Research, vol. 13, no. 11, pp. 5206-5217, 2014.

[8] J. T. Bagdanoff, M. S. Donoviel, A. Nouraldeen et al., "Inhibition of sphingosine 1-phosphate lyase for the treatment of rheumatoid arthritis: discovery of $(E)-1-(4-((1 R, 2 \mathrm{~S}, 3 R)-1,2,3,4-$ tetrahydroxybutyl)-1H -imidazol-2-yl)ethanone oxime (LX2931) and (1R,2 S,3R)-1-(2-(Isoxazol-3-yl)-1H -imidazol-4-yl)butane1,2,3,4-tetraol (LX2932)," Journal of Medicinal Chemistry, vol. 53 , no. 24, pp. 8650-8662, 2010.

[9] D. Ronghui and M. Chao, "Research progress in treating rheumatoid arthritis with traditional Chinese medicine," Tianjin Pharmacy, vol. 25, no. 1, pp. 75-78, 2013.

[10] Y. Wenqin, W. Dingyu, L. Yuanzhong et al., "Immunomodulatory effects of tetrastigma hypoglaucum planch on rats with rheumatoid arthritis," Herald of Medicine, vol. 35, no. 2, pp. 141143,2016

[11] Guizhou Investigation Team of Chinese Herbal Medicine for War Preparedness, Chinese Herbal Medicine in Guizhou, vol. I, Guizhou Science and Technology Information Unit, Guiyang, China, 1972.

[12] X. Bin and Z. Jie, "Chemical constituents from the fruit of illicium simonii," China Modern Doctor, vol. 46, no. 17, pp. 35-36, 2008.

[13] J. Liu, X. Zhang, Y. Shi, Q. Zhang, Y. Ma, and J. Chen, "Studies on chemical constituents of Illicium simonsii," China Journal of Chinese Materia Medica, vol. 36, no. 10, pp. 1311-1315, 2011.

[14] Z. Benneng, R. Haixing, W. Zijian et al., "Ecology, geographical distribution, and toxicology test of wild star anise in Northwest Guizhou," Journal of Sichuan Normal University (Natural Science), vol. 3, pp. 134-136, 1987.

[15] H. Tianfeng, H. Qing, and X. Wenhao, "To explore the special efficiency medicine recurring the critical patient of 'octagonal Hui seed of open space' toxicosis," Guangming Journal of Chinese mudicine, vol. 23, no. 2, pp. 187-188, 2008. 
[16] I. Kouno, C. Iwamoto, Y. Kameda, T. Tanaka, and C.-S. Yang, "A New Triphenyl-Type Neolignan and a Biphenylneolignan from the Bark of Illicium simonsii," Chemical \& Pharmaceutical Bulletin, vol. 42, no. 1, pp. 112-114, 1994.

[17] D.-D. Wei, J.-S. Wang, Y. Zhang, and L.-Y. Kong, "A new phenylpropanoid glycoside from the fruits of Illicium simonsii," Chinese Journal of Natural Medicines, vol. 10, no. 1, pp. 20-23, 2012.

[18] M. Ping, L. Meisheng, and Z. Dongqing, "Recent progress of the association research on IL-6/IL-6R and rheumatoid arthritis," Immunological Journal, vol. 27, no. 4, pp. 355-360, 2011 (Chinese).

[19] W. Jun and L. Xiaofeng, "Effects of the combination of compound rhubarb powder and methotrexate on the expression of MMP-10 and IL-33 in rheumatoid arthritis rats," Immunological Journal, vol. 31, no. 1, pp. 41-46, 2013.

[20] Y. Liying, "The effects of Bitongling granules and its partial formulations on the induction of IL-10 and IFN- $\gamma$ and release of MMP-2 and -9 in CIA rat spleen lymphocytes," Chinese Journal of the Practical Chinese with Modern Medicine, vol. 20, no. 19, pp. 1661-1663, 2007.

[21] L. Peipei, X. Guoxiong, S. Shanshan et al., "Clinical manifestations and the main evaluation method on adjuvant-induced arthritis model in rats," Chinese Journal of Immunology, vol. 28, no. 05, pp. 453-457, 2012.

[22] I. B. McInnes and G. Schett, "Cytokines in the pathogenesis of rheumatoid arthritis," Nature Reviews Immunology, vol. 7, no. 6, pp. 429-442, 2007.

[23] E. W. Karlson, L. B. Chibnik, and S. S. Tworoger, "Biomarkers of inflammation and development of rheumatoid arthritis in women from two prospective cohort studies," Arthritis \& Rheumatism, vol. 60, no. 3, pp. 641-652, 2009.

[24] W. Zhiming, G. Jian, and H. Qingshui, "Levels of the soluble triggering receptor expressed on myeloid cells-1 and interleukin-17 in the serum of rheumatoid arthritis patients," Immunological Journal, vol. 28, no. 4, pp. 317-320, 2012.

[25] S. K. Lundy, S. Sarkar, L. A. Tesmer, and D. A. Fox, "Cells of the synovium in rheumatoid arthritis. T lymphocytes," Arthritis Research \& Therapy, vol. 9, no. 1, 2007.

[26] Y. Gu, X. Hu, C. Liu, X. Qv, and C. Xu, "Interleukin (IL)-17 promotes macrophages to produce IL-8, IL- 6 and tumour necrosis factor- $\alpha$ in aplastic anaemia," British Journal of Haematology, vol. 142, no. 1, pp. 109-114, 2008.

[27] S.-Y. Lee, S.-K. Kwok, H.-J. Son et al., "IL-17-mediated Bcl2 expression regulates survival of fibroblast-like synoviocytes in rheumatoid arthritis through STAT3 activation," Arthritis Research \& Therapy, vol. 15, no. 1, 2013.

[28] S. A. Jones, "Directing transition from innate to acquired immunity: defining a role for IL-6," The Journal of Immunology, vol. 175, no. 6, pp. 3463-3468, 2005.

[29] F. De Benedetti, "Targeting interleukin-6 in pediatric rheumatic diseases," Current Opinion in Rheumatology, vol. 21, no. 5, pp. 533-537, 2009. 


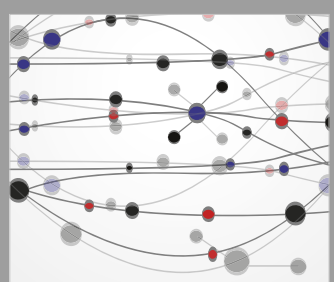

The Scientific World Journal
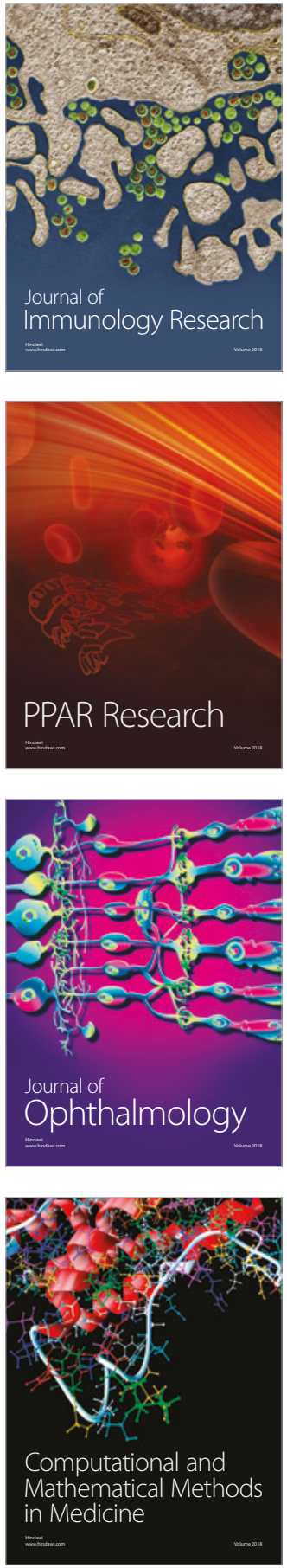

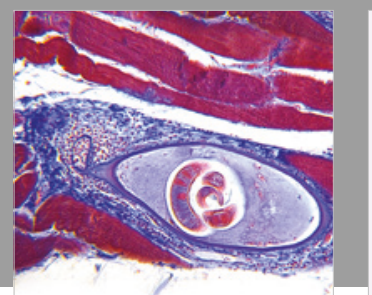

Gastroenterology Research and Practice

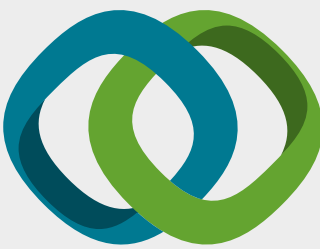

\section{Hindawi}

Submit your manuscripts at

www.hindawi.com
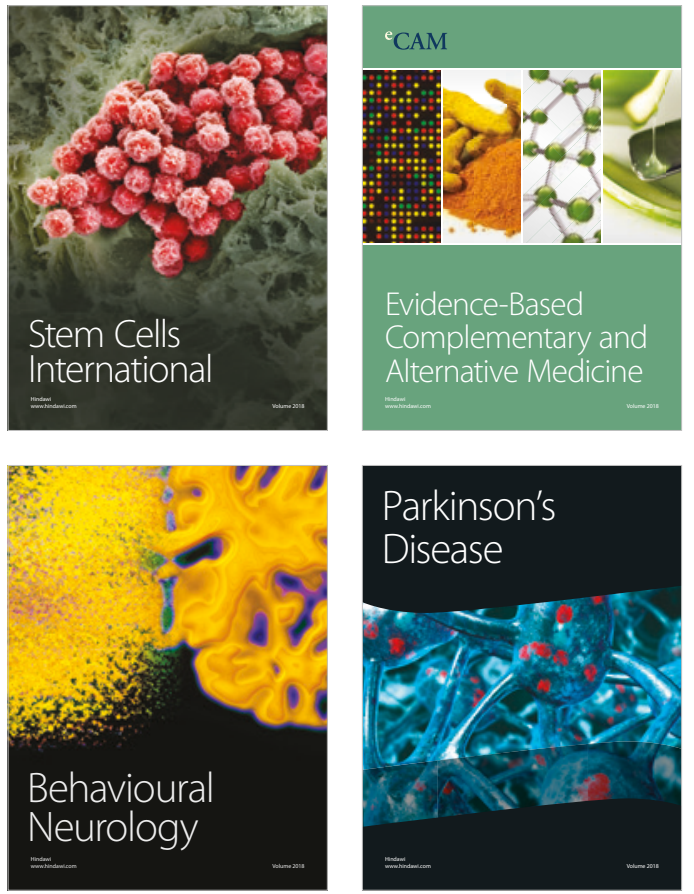

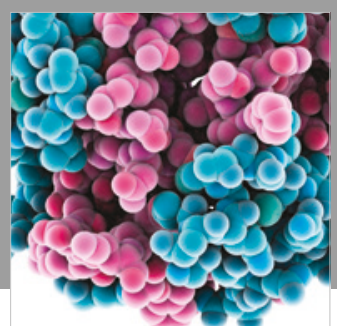

ournal of

Diabetes Research

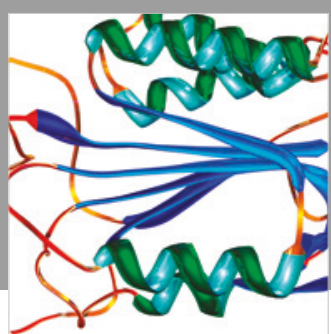

Disease Markers
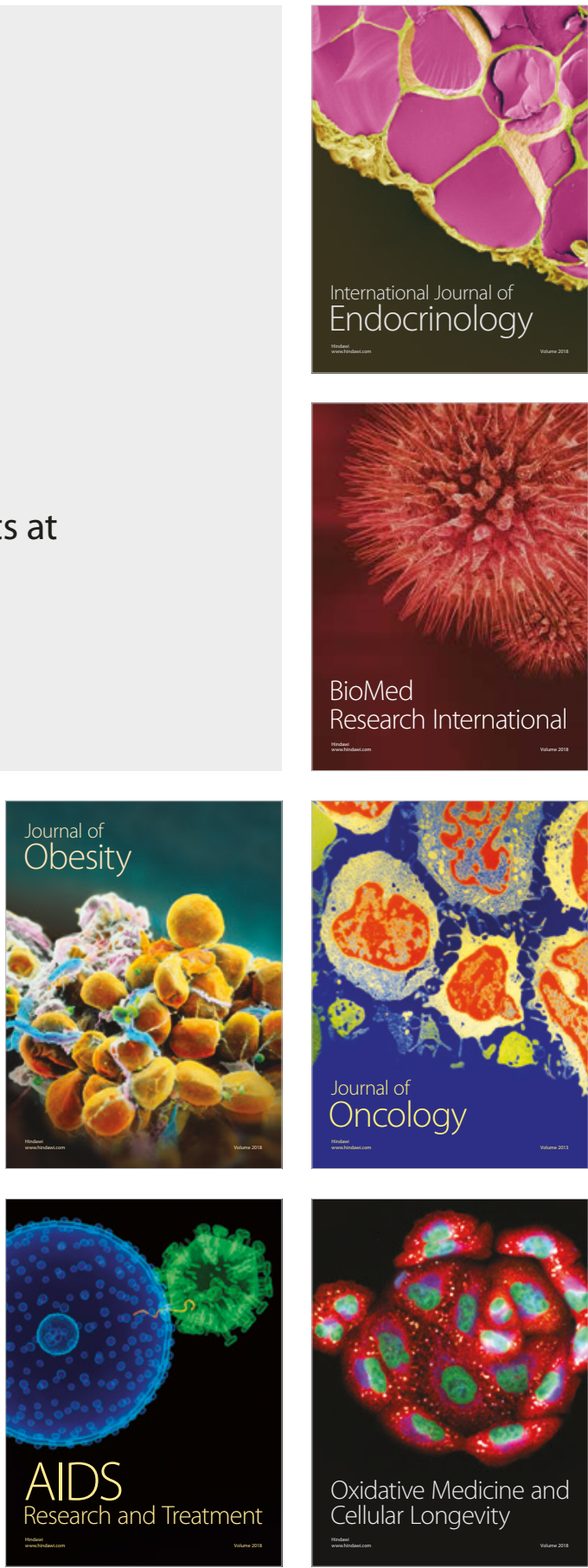